\title{
Conductas de riesgo sexual y reproductivo en estudiantes universitarios en Huancayo
}

\author{
Sexual and reproductive risk behaviour in Huancayo \\ college students
}

\author{
Claudia Ríos Cataño ${ }^{a}$ \\ a Universidad Continental
}

\section{RESUMEN}

El objetivo del estudio fue determinar las conductas de riesgo sexual y reproductivo en alumnos de una universidad particular. Se trata de un estudio, transversal, exploratorio y descriptivo, la muestra fue no probabilística conformada por 254 alumnos jóvenes; se utilizó el test Self-efficacy scale for AIDS (SEA27) que mide la autoeficacia en conductas de riesgo para el contagio de $\mathrm{VIH} / \mathrm{SIDA}$, así también se recolectaron datos sobre actitud sexual, conocimientos y adquisición de información en sexualidad; los datos fueron digitados, almacenados y analizados en el programa estadístico SPSS versión 22. Los resultados evidenciaron que, en cuanto a la autoeficacia para evitar conductas de riesgo el género masculino tuvo una autoeficacia promedio bajo de $43,6 \%$, mientras que el género femenino mostró tener una autoeficacia promedio alto 56,9 $\%$, en cuanto a las actitudes sexuales ambos sexos concuerdan que el hombre es el responsable por la protección en una relación sexual, siendo que el uso del preservativo es sólo cuando la mujer solicita, en relación a los conocimientos en sexualidad ambos sexos concuerdan que la condiciones de riesgo para contraer $\mathrm{VIH} / \mathrm{SIDA}$ es tener relaciones sin preservativo, tener relaciones sexuales con desconocidos y recibir transfusión de sangre. En conclusión los estudiantes universitarios a pesar de tener informaciones relativamente seguras y con miedos hacia el contagio, su conducta sexual es de riesgo, siendo que el género femenino presenta mayor autoeficacia en conductas de riesgo en relación al género masculino.

Palabras clave: Autoeficacia, síndrome de inmunodeficiencia, conducta de riesgo.

\section{ABSTRACT}

The aim was to determine the sexual and reproductive risk behaviour in Huancayo college students from a private institution. One is a research, cross-sectional, exploratory and descriptive, the sample nonprobabilistic was conform by 254 young students, the Self-efficacy test was used for AIDS (SEA27) that measures self-efficacy in risk behaviours for AIDS infect, thus also collected data on sexual attitude, knowledge and information acquisition in sexuality, data were digitates, stored and analyzed in SPSS statistical program. Results demonstrated about selfefficacy risk behaviours that masculine gender had one self-efficacy average under $43,6 \%$, whereas feminine gender showed to have one self-efficacy average high $56,9 \%$, also about sexual attitudes both sexes are agree with men's as the person in charge for the protection in a sexual relation, affirming that the use of the preservative only happen when the woman solicits, about sexuality knowledge both sexes are agree with the conditions of risk to contract AIDS is to have relations without preservative, to have sexual relations with strangers and to receive blood transfusions. In conclusion, the college students despite have information relatively safe and with fears towards the contagion, have sexual behaviours of risk, being feminine gender whose present greater self-efficacy against to gender male.

Keywords: Self-efficacy; AIDS; sexual behaviours of risk.

Historial del artículo:

Recibido, 14 de enero de 2016; aceptado, 10 de mayo de 2016; disponible en línea, 30 de junio de 2016

* Obstetriz, doctora en salud pública.

Correo: rcclaudita@gmail.com 


\section{INTRODUCCIÓN}

Históricamente la contaminación por Infecciones de Transmisión Sexual y Síndrome de Inmunodeficiencia Adquirida (ITS/SIDA) fueron asociadas a grupos de riesgo, como las minorías marginadas de homosexuales y usuarios de drogas, sin embargo en la actualidad con el aumento de casos se ha hecho necesario hablar de conductas de riesgo y no más grupos de riesgo, considerando que todas las personas sin excepción están sujetas a contaminarse, basta con tener comportamientos que envuelvan una situación de riesgo (1).

La incidencia de la infección por el Virus de Inmunodeficiencia Humana ( $\mathrm{VIH}$ ) en América Latina va en aumento, se estima que la mitad de los nuevos casos se dan en personas con edad inferior a 25 años que se han contaminado por vía sexual (1). Los jóvenes entre 15 a 24 años representan el $45 \%$ del total de nuevas infecciones por el $\mathrm{VIH}$, y gran cantidad de jóvenes aún no cuentan con información completa y exacta acerca de cómo evitar la exposición al virus (2).

Según el Ministerio de Salud (3), la información contradictoria, la intensa exposición a los mensajes estereotipados de los medios de comunicación, el debilitamiento del entorno familiar, la insuficiente o distorsionada información, la ausencia de valores orientadores, y el ejercicio no responsable de su sexualidad, lo podrían conducir a una infección por $\mathrm{VIH} / \mathrm{SIDA}$.

La mayoría de los adolescentes y jóvenes presentan déficit en el nivel de autoeficacia y susceptibilidad frente al VIH/SIDA, por las características propias de su edad, lo que desencadena la ejecución de comportamientos de riesgo para la infección por $\mathrm{VIH}$, y los convierte en una población vulnerable frente a esta infección (4).

Por lo tanto es necesario realizar estudios en la prevención desde el punto de vista de la autoeficacia, es decir adquirir o mantener conductas que eviten el contagio por $\mathrm{VIH}$. El concepto de autoeficacia se basa en la teoría sociocognitiva de Bandura, que hace referencia a la capacidad o convicción personal que tiene el sujeto de que pueda ejecutar exitosamente la conducta requerida para actuar o producir un resultado en una situación, actividad o dominio $(5,6)$.

Considerando que los adolescentes jóvenes son una población vulnerable la investigación tuvo como objetivo determinar las conductas de riesgo sexual y reproductivo en alumnos de una universidad privada del interior del Perú.

\section{MATERIAL Y MÉTODOS}

Se trata de un estudio no experimental, transversal, exploratorio y descriptivo, se realizó en una universidad privada de Huancayo, esta cuenta con las facultades de Ingeniería (1 1 especialidades), Ciencias de la empresa y humanidades (11 especialidades) y Ciencias de la salud (5 especialidades), cuenta con una población estudiantil promedio por semestre académico de 10 300 alumnos.

La muestra fue no probabilística conformada por 254 alumnos voluntarios de las facultades de ingeniería y ciencias de la empresa durante el año 2015; se optó por este tipo de muestra porque este tema demanda de honestidad e interés por responder a las informaciones solicitadas. Se tomó como criterio de exclusión a mayores de 24 años ya que el periodo de la juventud se ha definido entre 15 y 24 años (7), también fueron excluidos los alumnos de la facultad de ciencias de la salud por sus conocimientos previos relacionados con el tema ya que este conocimiento puede distorsionar los resultados, asimismo fueron excluidos alumnos casados o convivientes.

Se recolectaron los datos a través de un cuestionario que constaba de cuatro partes: la primera con preguntas sobre características generales del alumno; la segunda se utilizó para el test Self-Efficacy scale for AIDS (SEA 27) que mide la autoeficacia en conductas de riesgo para el contagio del VIH/SIDA (5); la tercera relacionada con la actitud sexual, y la cuarta parte referente con sus conocimientos y adquisición de información sobre sexualidad.

El test SEA 27 ha sido adaptado para la realidad latinoamericana, tiene validez demostrada y una confiabilidad por alfa de Cronbach de 0,92, mide la capacidad de evitar conductas de riesgo y tomar las medidas de seguridad adecuadas para prevenir el SIDA. El SEA 27 cuenta con 27 reactivos, que cuantifican cuatro factores de autoeficacia:

Factor 1: capacidad percibida para decir no a tener relaciones sexuales bajo diferentes circunstancias.

Factor 2: Autoeficacia para usar el preservativo.

Factor 3: Autoeficacia para preguntar al compañero sobre las relaciones sexuales anteriores y otras conductas de riesgo como consumo de drogas.

Factor 4: Autoeficacia para mantenerse virgen hasta el matrimonio, la fidelidad y el hablar de relaciones sexuales con los padres.

El SEA 27 es medida a través de una escala tipo Likert con opciones: nada seguro, algo seguro, medio seguro, muy seguro, y totalmente seguro. Para la 
cuantificación se tiene una puntuación directa para cada uno de los factores, siendo su interpretación como: muy bajo nivel de autoeficacia, bajo, promedio bajo, promedio alto, alto y muy alto, en puntuaciones directas para cada factor diferenciadas por género.

Para evaluar la actitud hacia el uso de preservativo, la planificación de la relación sexual, la monogamia, y conducta sexual (segunda parte del cuestionario), consta de 10 variables, aplicadas con una escala tipo Likert con opciones: nada seguro, algo seguro, medio seguro, muy seguro, y totalmente seguro.

Los datos fueron analizados con el programa estadístico SPSS versión 22. Para el análisis estadístico se utilizó la con una desviación estándar de 2,4 y una moda de 19. En cuanto a la religión los alumnos refirieron ser católicos $77,6 \%$ (194), otras religiones 7,0 \% (18) y se abstuvieron de dar respuesta 15,4\% (39).

En relación a la autoeficacia para evitar las conducta de riesgo para el contagio de $\mathrm{VIH}$ a partir de una puntuación directa total para el género masculino, se mostró un nivel de autoeficacia muy bajo 1,7 \% (2), bajo $9,4 \%(11)$, promedio bajo $43,6 \%$ (51), promedio alto $34,2 \%(40)$ y alto $11,1 \%(13)$, se aprecia que el que el mayor porcentaje se encuentra con una autoeficacia promedio, y cabe destacar que no se encontró a ningún entrevistado con un nivel muy alto de autoeficacia.

Tabla $\mathrm{N}^{\circ}$ 1: Nivel de autoeficacia para evitar conductas de riesgo para el contagio con VIH según sus cuatro factores, en alumnos universitarios de género masculino, 2015.

\begin{tabular}{|c|c|c|c|c|c|c|c|c|}
\hline \multirow{3}{*}{ Clasificación } & \multicolumn{8}{|c|}{ Autoeficacia para evitar conductas de riesgo según factores } \\
\hline & \multicolumn{2}{|c|}{ Factor 1} & \multicolumn{2}{|c|}{ Factor 2} & \multicolumn{2}{|c|}{ Factor 3} & \multicolumn{2}{|c|}{ Factor 4} \\
\hline & $\mathrm{n}$ & $\%$ & $\mathrm{n}$ & $\%$ & $\mathrm{n}$ & $\%$ & $\mathrm{n}$ & $\%$ \\
\hline Muy bajo & 17 & 14,5 & 5 & 4,3 & 2 & 1,7 & 39 & 33,3 \\
\hline Bajo & 23 & 19,7 & 18 & 15,4 & 11 & 9,4 & 37 & 31,6 \\
\hline Promedio bajo & 22 & 18,8 & 31 & 26,5 & 39 & 33,3 & 16 & 13,7 \\
\hline Promedio alto & 37 & 31,6 & 27 & 23,1 & 38 & 32,5 & 17 & 15,4 \\
\hline Alto & 12 & 10,3 & 20 & 17,1 & 12 & 10,3 & 5 & 4,3 \\
\hline Muy alto & 6 & 5,1 & 16 & 13,7 & 15 & 12,8 & 3 & 2,6 \\
\hline Total & 117 & 100,0 & 117 & 100,0 & 117 & 100,0 & 117 & 100,0 \\
\hline
\end{tabular}

Tabla $N^{\circ}$ 2: Nivel de autoeficacia para evitar conductas de riesgo para el contagio con $\mathrm{VIH}$ según sus cuatro factores, en alumnos universitarios de género femenino, 2015.

\begin{tabular}{|c|c|c|c|c|c|c|c|c|}
\hline \multirow{3}{*}{ Clasificación } & \multicolumn{8}{|c|}{ Autoeficacia para evitar conductas de riesgo según factores } \\
\hline & \multicolumn{2}{|c|}{ Factor 1} & \multicolumn{2}{|c|}{ Factor 2} & \multicolumn{2}{|c|}{ Factor 3} & \multicolumn{2}{|c|}{ Factor 4} \\
\hline & $\mathrm{n}$ & $\%$ & $\mathrm{n}$ & $\%$ & $\mathrm{n}$ & $\%$ & $\mathrm{n}$ & $\%$ \\
\hline Muy bajo & 2 & 2,9 & 4 & 2,9 & 1 & 0,7 & 34 & 24,8 \\
\hline Bajo & 8 & 5,8 & 6 & 4,4 & 5 & 3,6 & 39 & 28,5 \\
\hline Promedio bajo & 48 & 35,0 & 28 & 20,4 & 32 & 23,4 & 24 & 17,5 \\
\hline Promedio alto & 55 & 40,0 & 36 & 26,3 & 43 & 31,4 & 31 & 22,6 \\
\hline Alto & 16 & 11,7 & 38 & 27,7 & 31 & 22,6 & 5 & 3,6 \\
\hline Muy alto & 6 & 4,4 & 25 & 18,2 & 25 & 18,2 & 4 & 2,9 \\
\hline Total & 137 & 100,0 & 137 & 100,0 & 137 & 100,0 & 137 & 100,0 \\
\hline
\end{tabular}

estadística descriptiva del tipo de promedio, desviación estándar y porcentajes. Para identificar la asociación entre las variables cualitativas se utilizó la prueba de la Ji cuadrada, con un nivel de significancia de 0,05.

\section{RESULTADOS}

En cuanto a las características del alumno 45,2\% (117) fueron de sexo masculino y de sexo femenino $54,8 \%$ (139). En cuanto a la facultad el 76,4 \% (194) pertenecían a la facultad de Ciencias de Empresa y Humanidades, mientras que el $23,6 \%(60)$ eran de la facultad de Ingeniería. La edad media fue de 19,8,
En cuanto a la autoeficacia para evitar conductas de riesgo según los cuatro factores para el género masculino (tabla 1) se evidencia que el factor 1 la mayoría puntuó como una autoeficacia promedio; para el factor 2 se encontró con un promedio bajo y promedio alto $49,6 \%$ (58), para el factor 3 la mayoría puntuó promedio bajo y promedio alto $65,8 \%$ (77), para el factor 4 se destaca que el mayor porcentaje se encuentra en muy bajo y bajo $64,9 \%$ (76) lo que se traduce en la incapacidad para mantenerse virgen, así como practicar la fidelidad y conversar sobre sexo con sus padres para el género masculino.

En relación a la autoeficacia para evitar las conducta de riesgo para el contagio de $\mathrm{VIH}$ a partir de una 
puntuación directa total para el género femenino, se muestra un nivel de autoeficacia bajo de 2,2 \% (3), promedio bajo $25,5 \%$ (35), promedio alto $56,9 \%$ (78) y alto $15,3 \%(21)$, se aprecia que el mayor porcentaje se encuentra con una autoeficacia promedio alto, tampoco se encontró a ningún participante en los extremos de la escala (muy bajo, muy alto nivel de autoeficacia). En cuanto a la autoeficacia para evitar conductas de riesgo según los cuatro factores para el género femenino (tabla 2) se evidencia que para el factor 1 la mayoría puntuó como una autoeficacia promedio 75,0\% (103); para el factor 2 se encontró en promedio alto y alto $54 \%$ (74), para el factor 3 la mayoría puntuó promedio bajo y promedio alto $54,8 \%$ (75), para el factor 4 se destaca que el mayor porcentaje se encuentra en muy bajo y bajo 53,3\% (73) lo que se traduce en la incapacidad a mantenerse virgen, así como practicar la fidelidad y conversar sobre sexo con sus padres.

Tabla $N^{\circ}$ 3: Actitud sexual, uso de preservativo e información sobre sexualidad en alumnos universitarios de género masculino, 2014.

\begin{tabular}{|c|c|c|c|c|c|c|c|c|c|c|c|}
\hline \multirow{2}{*}{$\begin{array}{c}\text { Actitud } \\
\text { sexual }\end{array}$} & \multicolumn{2}{|c|}{$\begin{array}{l}\text { Nada } \\
\text { Seguro } \\
\text { n } \% \\
\end{array}$} & \multicolumn{2}{|c|}{$\begin{array}{c}\text { Algo } \\
\text { seguro } \\
\text { n } \% \\
\end{array}$} & \multicolumn{2}{|c|}{$\begin{array}{l}\text { Medio } \\
\text { seguro } \\
\text { n } \%\end{array}$} & \multicolumn{2}{|c|}{$\begin{array}{c}\text { Muy } \\
\text { seguro } \\
\text { n } \%\end{array}$} & \multicolumn{2}{|c|}{$\begin{array}{c}\text { Totalmente } \\
\text { seguro } \\
\text { n } \%\end{array}$} & \multirow{2}{*}{$\frac{n}{117}$} \\
\hline & 21 & 17,9 & 17 & 14,5 & 38 & 32,5 & 22 & 18,8 & 19 & 16,2 & \\
\hline A2 & 17 & 14,5 & 9 & 7,7 & 30 & 25,6 & 35 & 29,9 & 26 & 22,2 & 117 \\
\hline A3 & 25 & 21,4 & 18 & 15,4 & 34 & 29,1 & 22 & 18,8 & 18 & 15,4 & 117 \\
\hline A4 & 19 & 16,2 & 16 & 13,7 & 43 & 36,8 & 19 & 16,2 & 20 & 17,1 & 117 \\
\hline A5 & 10 & 8,5 & 20 & 17,1 & 33 & 28,2 & 29 & 24,8 & 25 & 21,4 & 117 \\
\hline A6 & 6 & 5,1 & 9 & 7,7 & 17 & 14,5 & 40 & 34,2 & 45 & 38,5 & 117 \\
\hline A7 & 30 & 25,6 & 23 & 19,7 & 30 & 25,6 & 21 & 17,9 & 13 & 11,1 & 117 \\
\hline A8 & 63 & 53,8 & 16 & 13,7 & 22 & 18,8 & 6 & 5,1 & 10 & 8,5 & 117 \\
\hline A9 & 57 & 48,7 & 25 & 21,4 & 19 & 16,2 & 11 & 9,4 & 5 & 4,3 & 117 \\
\hline $\mathrm{A} 10$ & 8 & 6,8 & 11 & 9,4 & 43 & 36,8 & 32 & 27,4 & 33 & 19,7 & 117 \\
\hline
\end{tabular}

Estás de acuerdo con que .... A1: la mujer es la que debe solicitar el preservativo; $\mathrm{A2}$ : el hombre debe comprar los preservativos; A3: el hombre es el responsable por la protección durante la relación sexual; A4: la mujer debe traer los preservativos; A5: las relaciones sexuales deben ser espontáneas; $A 6$ : tu actual situación te permite comprar preservativo; A7: las relaciones sexuales deben de tenerse con la misma persona de por vida; A8 prefiero arriesgarme a contraer una enfermedad, a no tener relaciones sexuales; A9: el SIDA es algo muy difícil de adquirir aunque no me proteja; A10: consideras que la información que tienes sobre sexualidad es.

Tabla $N^{\circ}$ 4: Actitud sexual, uso de preservativo e información sobre sexualidad en alumnos universitarios de género femenino, 2014.

\begin{tabular}{|c|c|c|c|c|c|c|c|c|c|c|c|}
\hline \multirow{2}{*}{$\begin{array}{c}\begin{array}{c}\text { Actitud } \\
\text { sexual }\end{array} \\
\mathrm{Al}\end{array}$} & \multicolumn{2}{|c|}{$\begin{array}{l}\text { Nada } \\
\text { Seguro } \\
\text { n } \%\end{array}$} & \multicolumn{2}{|c|}{$\begin{array}{c}\text { Algo } \\
\text { seguro } \\
\text { n } \quad \%\end{array}$} & \multicolumn{2}{|c|}{$\begin{array}{l}\text { Medio } \\
\text { seguro } \\
\mathrm{n} \quad \%\end{array}$} & \multicolumn{2}{|c|}{$\begin{array}{c}\text { Muy } \\
\text { seguro } \\
\text { n } \quad \%\end{array}$} & \multicolumn{2}{|c|}{$\begin{array}{c}\text { Totalmente } \\
\text { seguro } \\
\text { n } \%\end{array}$} & \multirow{2}{*}{$\frac{\mathrm{n}}{137}$} \\
\hline & 21 & 15,3 & 14 & 10,2 & 47 & 34,3 & 26 & 19,0 & 29 & 21,2 & \\
\hline A2 & 9 & 6,6 & 8 & 5,8 & 40 & 29,2 & 33 & 24,1 & 47 & 34,3 & 137 \\
\hline A3 & 25 & 18,2 & 24 & 17,5 & 42 & 30,7 & 22 & 16,1 & 24 & 17,5 & 137 \\
\hline A4 & 29 & 21,2 & 36 & 26,3 & 44 & 32,1 & 17 & 12,4 & 11 & 8,0 & 137 \\
\hline A5 & 28 & 20,4 & 21 & 15,3 & 40 & 29,2 & 28 & 20,4 & 20 & 16,6 & 137 \\
\hline A6 & 6 & 4,4 & 3 & 2,2 & 14 & 10,2 & 46 & 33,6 & 68 & 49,6 & 137 \\
\hline A7 & 10 & 7,3 & 13 & 9,5 & 29 & 21,2 & 42 & 30,7 & 43 & 31,4 & 137 \\
\hline A8 & 74 & 54,0 & 15 & 10,9 & 24 & 17,5 & 14 & 10,2 & 10 & 7,3 & 137 \\
\hline A9 & 73 & 53,3 & 11 & 8,0 & 35 & 25,5 & 7 & 5,1 & 11 & 8,0 & 137 \\
\hline $\mathrm{A} 10$ & 4 & 2,9 & 10 & 7,3 & 49 & 35,8 & 42 & 30,7 & 32 & 23,4 & 137 \\
\hline
\end{tabular}

Estás de acuerdo con que .... A1: la mujer es la que debe solicitar el preservativo; A2: el hombre debe comprar los preservativos; A3: el hombre es el responsable por la protección durante la relación sexual; A4: la mujer debe traer los preservativos; A5: las relaciones sexuales deben ser espontáneas; A6: tu actual situación te permite comprar preservativo; A7: las relaciones sexuales deben de tenerse con la misma persona de por vida; A8 prefiero arriesgarme a contraer una enfermedad, a no tener relaciones sexuales; A9: el SIDA es algo muy difícil de adquirir aunque no me proteja; A10: consideras que la información que tienes sobre sexualidad es. 
En relación a la escala sobre actitud sexual y uso del preservativo para el género masculino (tabla 3) se evidencia en la primera variable (la mujer debe solicitar los preservativos) los participantes $35 \%$ (41) están de acuerdo con esta afirmación, otra variable que llama la atención es la actitud A2 (estás de acuerdo con que el hombre es el responsable por la protección durante la relación sexual) la mayoría 52,1 \% (51) concuerdan con esta afirmación. Otra actitud que se destaca es la A6 (estás de acuerdo que tu actual situación te permite comprar preservativos) siendo que la mayoría $72 \%$ (85) tiene las condiciones económicas de adquirirlo.

En cuanto a la actitud A8 (estás de acuerdo con que prefiero arriesgarme a contraer una enfermedad a no tener relaciones sexuales) la mayoría $67,5 \%$ (79) afirma que no se siente seguro con esa proposición aunque también se encuentra un pequeño porcentaje $13,6 \%$ (16) que están seguros de arriesgarse y no perder la oportunidad de tener relaciones sexuales, en cuanto a la variable A9 (el SIDA es difícil de contraer aunque no me proteja) la mayoría 70,1 \% (82) se mostró nada seguro en relación a esta afirmación. Por ultimo A10 (consideras que la información que tienes sobre sexualidad es) la mayoría 54, 1 \% (74) concuerdan que sus informaciones son seguras.

En relación a la escala sobre actitud sexual y uso del preservativo para el género femenino (tabla 4) se evidencia en la primera variable (la mujer debe solicitar los preservativos) los participantes 34,2 \% (47) se encuentran medio seguro, en cuanto a la segunda variable A2 (estás de acuerdo con que el hombre debe comprar los preservativos) la mayoría 58,4\% (80) concuerdan con esta afirmación. Otra actitud que se destaca es la A6 (estás de acuerdo que tu actual situación te permite comprar preservativos) siendo que la mayoría $62,1 \%$ (85) tiene las condiciones de adquirirlo, para la variable A7 (estás de acuerdo que las relaciones sexuales deben ser con la misma persona de por vida) la mayoría 62,1 \% (85) están seguras de esta afirmación. Las respuestas del A8 y A9 evidencian el miedo al contagio de una enfermedad de transmisión sexual. Por último la mayoría 54,1\% (74) concuerda en que sus informaciones en sexualidad son seguras.

En cuanto al inicio de su actividad sexual para el género masculino el $80,3 \%$ (94) manifestaron haber iniciado su vida sexual y que la primera relación sexual usaron preservativos en el $50 \%$ (47) y el resto no se protegió. El cuanto el género femenino el 51,1 \% (70) manifestaron haber iniciado su vida sexual y que en su primera relación sexual utilizaron preservativo el 29,2 $\%$ (40).

En relación a los conocimientos sobre sexualidad se les indicó que mencionen tres condiciones de riesgo para contraer VIH/SIDA, las respuestas más frecuentes del total de participantes fueron tener relaciones $\sin$ preservativo $65,4 \%(116)$, tener relaciones sexuales con desconocidos $46,9 \%$ (119) y recibir transfusión de sangre $41,3 \%$ (105), entre tanto las respuestas menos frecuentes fueron tener relaciones sexuales bajo el efecto del alcohol 11,8\% (30), ser infiel 10,6\% (27) y tener relaciones sexuales estando drogado $5,1 \%$ (13).

Asímismo se les solicitó mencionar tres fuentes de información donde han adquirido información sobre sexualidad en el caso del género masculino respondió en el internet $67,5 \%(79)$, escuela $53 \%$ (62) y libros $29,1 \%$ (34); entretanto el género femenino respondió $61,3 \%(84)$ en la escuela 48,9\% (67) en el internet y 43,1 \% (59)con la mamá.

Por otro lado se relacionó el nivel de autoeficacia y el uso de preservativos en la primera relación sexual, también la edad e inicio de la vida sexual entre otros pero en ningún caso se mostró estadísticamente significativo.

\section{DISCUSIÓN}

En un estudio de Ferreira (8), se demostró que las tendencias del comportamiento sexual de los jóvenes adolescentes son más positivos que los últimos años, pues hacen uso de métodos anticonceptivos y le dan importancia a la práctica del sexo seguro, los adolescentes saben que deben usar el preservativo pero en muchos casos no lo hacen, estos resultados concuerdan con los hallazgos, que evidenciaron un nivel de autoeficacia promedio ambos géneros, así también el factor 4 de autoeficacia se presentó un nivel bajo y muy bajo. En cuanto a las actitudes ambos géneros coinciden en tener las posibilidades de comprar preservativos sin embargo consideran que la responsabilidad recae en el género masculino, ambos géneros coinciden que es muy fácil contraer algún ITS y el miedo al tener relaciones sexuales en situaciones de riesgo.

Lumbrera (9) evidenció que el $44 \%$ de los estudiantes habían iniciado su vida sexual y la mayoría no planeó su primera relación sexual, sin embargo, más de la mitad (55\%) utilizó condón, lo cual concuerda con los resultados del presente estudio, a pesar de los miedos que tienen los alumnos respecto al contagio de $\mathrm{VIH} /$ SIDA, saben de la importancia del uso de preservativos, los alumnos manifestaron haber tenido su primera relación sexual sin protección; asimismo Uribe (4), demostró que los conocimientos no coinciden con la conducta sexual y la protección ante el $\mathrm{VIH}$, debido a que continúan teniendo relaciones sexuales sin protección, a pesar de percibir el riesgo de adquirir esta infección.

En el caso de las fuentes de información sobre sexualidad el género femenino refirió obtener información con 
sus padres específicamente con la madre lo que se ve como algo positivo pero hay que destacar que según Bárcena (10) demostró que las creencias negativas de los hijos en relación a sexualidad se incrementaron cuando los padres tuvieron mayor nivel de estereotipos hacia el cuidado de la salud sexual de sus hijos, demostrando que las creencias de los padres afectan a las enseñanzas que transmiten a los hijos, siendo importante una capacitación también para los padres de familia.

En el presente estudio ambos géneros coincidieron que la primera fuente de información en sexualidad es el Internet y lo fue en los colegios, lo que coincide con el estudio de Varela (11), donde se evidenció que las informaciones proceden del Internet $(30,8 \%)$, la información recibida en el centro escolar para el $38,6 \%$ de los encuestados, así como coincidió que el género femenino se informaba con la madre en $55 \%$, se destaca la necesidad como universidad de ofrecer información confiable ya que el Internet puede llevar a falsos mitos e prácticas inadecuadas para quien recién explora su sexualidad.

El estudio evidencia que los alumnos tiene un miedo a contagiarse con alguna ITS, saben de los cuidados, pero esto no es demostrado con sus comportamientos, al igual que el género femenino coincide que las relaciones sexuales deben ser con una pareja para toda la vida, sin embargo obtuvieron autoeficacia baja y muy baja cuando se hablaba de fidelidad y de mantenerse virgen hasta el matrimonio, lo cual muestra una limitación en esta clase de trabajo, según Meza-Gallardo (12), menciona que la presión ejercida por padres y educadores, puede influir en que las respuestas que no sean del todo sinceras, aunque los varones tienden a declarar su conducta sexual, al contrario del género femenino.

Las conclusiones son:

Los estudiantes universitarios a pesar de tener informaciones relativamente seguras y con miedos hacia el contagio, su conducta sexual es de riesgo, siendo que el género femenino presenta mayor autoeficacia en relación al género masculino. también queda claro que los alumnos tienen conocimientos parciales ciertos puntos específicos sobre su salud sexual, por lo tanto es necesario promover y promocionar actividades educativas en este tema tanto para los alumnos como para los padres que en muchos casos son su fuente de información.

Conflictos de interés: la autora declara no tener conflictos de interés con la publicación de este artículo.

\section{REFERENCIAS BIBLIOGRÁFICAS}

1. Camara SG, Sariera SC, Carlotto MS. Predictores de conductas sexuales de riesgo ante adolescentes. Interam J Psychol. 2007;41(2): 161-6.

2. Uribe AF, Orcasita LT, VélezTV. Factores de riesgo para la infección por VIH/SIDA en adolescentes y jóvenes colombiano. Act Colomb Psicol. 2010;13(1):11-24.

3. Ministerio de Salud [Internet].Perú. Salud sexual y reproductiva. [citado 2015 oct 20]. Disponible en: http://www.minsa.gob.pe/portada/est_san/ saludsexual.htm

4. Uribe RAI, Vélez TV, Barona C. Susceptibilidad y autoeficacia frente al VIH/Sida en adolescentes de Cali-Colombia. Rev Latinoam cienc soc niñez juv.2009;47(2) suppl 1:1513-33.

5. Lopez-Rosales F, Moral de la Rubia J. Validación de una escala de autoeficacia para la prevención del SIDA en adolescentes. Salud pública de Mexico. 2005;43(3):421-432.

6. Palacios DJR. Estimación psicométrica de la escala de autoeficacia ante conductas de riesgo para adolescentes en México. Psychosocial Interventions. 2015;24(1):1-7

7. Bahamón MMJ, Viancho PMA, Tobos VAR. Prácticas y conductas sexuales de riesgo en jóvenes: una perspectiva de género. 2014;31(2):327-53.

8. Ferreira MMSRS, Torgal MCIFPR. Estilos de vida en la adolescencia; comportamiento sexual de los adolescentes portugueses. Rev Esc Enferm USP. $2011 ; 45(3): 589-95$.

9. Lumbrera DI, Montezuma AG, Dosamantes $\mathrm{CL}$, Medina HMA, Cervantes RM, Lopez LMR. Estilos de vida y riesgos para la salud en estudiantes. Rev digital universitaria.2009;10(2):1-14 .

10. Bárcena GS, Robles MS, Diaz-Loving R. El papel de los padres en la salud de sus hijos. Acta de investigación psicol. 2013;3(1):956-68.

11. Varela SM, Paz EJ. Estudio sobre conocimientos y actitudes sexuales en adolescentes y jóvenes. Rev Int Androl. 2010;8(2):74-80.

12. Meza-Gallardo MI, Barella-Balboa JL, CobeñaManzorro M. Comportamientos y uso del preservativo en adolescentes de nuestro entorno. Aten Primaria. 2004;33(7):374-80. 